\title{
Psoas abscess as the first manifestation of Crohn's disease - case report
}

\author{
HARRY NIKOLIĆ ${ }^{1}$, ANA BOSAK VERŠIĆ ${ }^{1}$, IVICA ĆEPIĆ ${ }^{2}$ DAMIR HASANDIĆ ${ }^{1}, S^{2}$ DZA- \\ NA SRŠEN MEDANČIĆ ${ }^{1}$, DAMIR MILETIC ${ }^{3}$, TOMISLAV TADIN ${ }^{4}$, MLADEN PERŠIĆ
}

1 Pediatric Surgery Department, University Hospital Rijeka, Croatia

2 General Surgery Department, University Hospital Rijeka, Croatia

3 Department of Radiology, University Hospital Rijeka, Croatia

4 Ultrasound Diagnostic Service, Health Centre Rijeka, Croatia

5 Pediatrics Department, University Hospital Rijeka, Croatia

\author{
Corresponding author: \\ Tomislav Tadin \\ Ultrasound Diagnostic Service \\ Martina Kontuša 18 \\ 51000 Rijeka \\ Croatia \\ Phone: 00385959062464 \\ E-mail: medicina_ri@inet.hr
}

\section{ABSTRACT}

A case of 16-year-old female with secondary psoas abscess as the complication of the Crohn's disease was analysed. Her pre-hospital, long lasting, non-specific symptoms led to ultrasound (US) and radiologic imaging confirming the diagnosis. The antibiotic treatment was insufficient and worsening of the patient's condition required surgical treatment including descendent colectomy, abscess drainage and negative pressure wound therapy (NPWT). In spite of numerous complications the condition of the child gradually improved and she was discharged from the hospital on the 71 st postoperative day. Two years after the colectomy, the occlusion of her colostomy was performed.

Key words: child, Crohn's disease, psoas abscess

\section{INTRODUCTION}

Psoas abscess (PA) is an extremely rare pathology in children, particularly as complication of the Crohn's disease (CD). $(1,2)$ With its non-specific symptoms and insidious course, PA is often diagnosed late and with late adequate treatment. The primary PA (caused by haematogenic or lymphatic spread from remote locations) should be distinguished from the secondary PA (direct spread of the infect from nearby tissues). (2) The primary PA predominates in undeveloped countries and in children, while the secondary PA predominates in

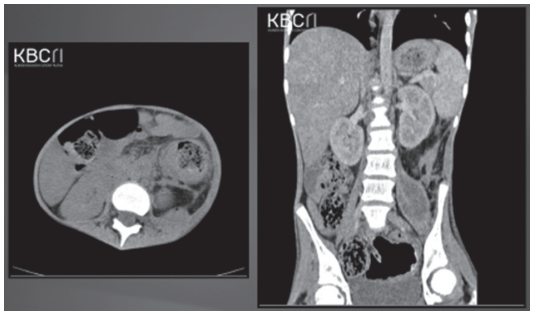

Figure 1. Abdominal computed tomographyscan

developed countries and in adults. (3) The most common cause of the secondary PA is CD. (4) A 16-year-old female with PA as the first symptom of $\mathrm{CD}$ was analysed. $\mathrm{Pa}$ tient's $\mathrm{CD}$ had not been diagnosed before.

\section{CASE REPORT}

At the age of 14 , the patient underwent evaluation due to delay of growth and anaemia of uncertain cause. Growth hormone therapy and iron supplementation were administered. Until the age of 16 , she had no other health issues. Two months before her admittance to the hospital, she began complaining of abdominal pain and diarrhoea without blood or mucus in her stool, loosing $8 \mathrm{~kg}$ during that period. A week prior to admittance, she began feeling pain in her left hip and leg with periodical fever. Physical examination revealed left lower abdomen tenderness and the left psoas sign was positive. Laboratory findings showed inflammatory changes. Stool tests for parasites, bacteria, viruses and blood and haemocultures with tubercu-

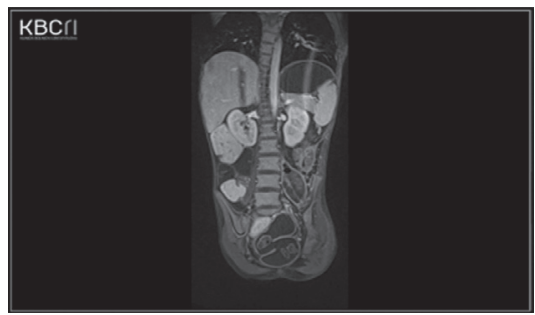

Figure 2. Abdominal magnetic resonance imiging scan

losis assay were negative. US revealed oedematous and hyperaemic bowel in left hemiabdomen with no peristalsis and with psoas collection which was confirmed by abdominal computerised tomography (CT) imaging (figure 1) and magnetic resonance imaging (MRI) enterocolonography (figure 2).

Antibiotic therapy was administered. The punction of the abscess with microbial analysis revealed Enterococcus faecalis and Escherichia coli. Antibiotic therapy was continued with complete enteral $\mathrm{CD}$ nutrition. The patient's general condition was satisfactory with periodical subfebrility, mild abdominal tenderness and elevated inflammatory markers. On the 10th day high fever, severe meteorism and abdominal colicae developed. US detected increased diameter of the left iliopsoas collection. The descendent colectomy and evacuation of the psoas abscess were performed, with drainage and colostomy. Due to oedematous and dilated small intestines and the extremely narrow and small pelvic cavity (due to her delay of growth), the abdominal wall closure was impossible, so 
negative pressure wound therapy (NPWT) was applied. Histopathological analysis revealed chronic inflammatory bowel disease (IBD). Antibiotic and supportive therapy was continued. Upon the first NPWT change, duodenal perforation was detected and drained with Nelaton catheter through the perforation hole to the abdominal wall. On the 7th postoperative day oesophagogastroduodenoscopy confirmed correct location of the Nelaton catheter and the nasojejunal Frekka triluminal catheter for feeding was placed in the distal duodenum. On the 10th postoperative day the patient, with improved general condition, was extubated, NPWT was removed and the abdominal wall was closed. Complete enteral nutrition was introduced. Duodenal catheter was removed six weeks after the operation. During the hospital treatment the left peroneal nerve palsy developed. Continuous physical therapy was applied. The patient was discharged from the hospital on the 71st postoperative day. During the following nine months the patient's general condition gradually improved, as well as her peroneal palsy. Two years after the colectomy the occlusion of her colostomy was performed.

\section{DISCUSSION}

$\mathrm{IBD}$, especially $\mathrm{CD}$, is the most common secondary PA cause due to well irrigated iliopsoas. (1-4) This complication happens rarely according to Veroux and associates (0.9\% incidence). (1) Agha and associates reported that in $26.5 \%$ of $\mathrm{CD}$ patients the first manifestation was PA. None of their patients younger than 1 year had this complication. (5) This clearly states that PA complicating CD in children happens extremely seldom. (1-4) Nonspecific symptoms complicate the diagnostic process and postpone the therapy. Classical trias of PA symptoms: back pain, limp and fever can be seen in only $30 \%$ of patients. (6) The dominating symptoms in our patient were pain in her left hip and leg, limping, significant weight loss, fatigue, positive psoas sign and tender abdominal mass in her left lower quadrant. Her periodical diarrhoea, nonspecific abdominal pain and periodical fever were interpreted as CD symptoms and, furthermore, we believe that her first CD symptom was delay of growth that had been previously regarded as growth hormone deficiency. Her anaemia was also identified as $\mathrm{CD}$ symptom since no menarche was reported. Increased inflammatory markers, anaemia and low serum albumin levels can be found in $\mathrm{CD}$ patients. (6) Our patient had all these laboratory changes. Haemoculture is positive in only $75 \%$ of PA patients. The most common pathogens are Staphylococcus aureus (primary PA), Escherichia coli and rarely Streptococcus viridans, Enterococcus faecalis, Bacteroides species, Yersinia enterocolitica, Clostridium (secondary PA). (7) In our patient the haemocultures were negative, while the punction of the abscess was positive for Enterococcus faecalis and Escherichia coli. US is diagnostically significant in only $60 \%$. (7) For the final diagnosis CT or MRI is obligatory, with MRI being more sensitive for diagnosing primary disease and its complications. (3) In our patient US pointed to possible abscess. Multislice CT set the PA diagnosis. MRI enterocolonography indicated that the PA was secondary, complicating CD. Although antibiotic treatment can be ef- fective enough for treating PA, incision and percutaneous drainage with antibiotic treatment represent correct initial therapy. $(1,4,8)$ However, if PA is a complication of $\mathrm{CD}$, this therapy is only preparation for operational treatment of affected bowel segment. (8) In our patient we administered antibiotic therapy. However, worsening of the general condition required urgent surgery, i.e. incision, evacuation and drainage of the abscess, resection of the descendent colon and colostomy. Due to impossibility of abdominal wall closure, NPWT therapy was applied. Despite numerous complications, her condition improved with intensive support therapy. Eighteen months after the surgery biological therapy was successfully applied. Two years after the operation the patient's colostomy was occluded. PA prognosis is still uncertain. Death rate is $10.5 \%$, mostly due to sepsis with general deterioration and malnutrition. (9) One of rare PA complications is lower extremity nerve palsy possibly due to its direct spread through the hip with the attendant occurrence of septic arthritis. (10) Our patient had left peroneal nerve palsy which improved after intense physical therapy.

\section{CONCLUSION}

IBD as the cause of PA should be thought of more often. That would prevent possible complications in both children and adults. Having this in mind, even though rare, CD should be considered as possible PA cause in children.

\section{REFERENCES}

1. Veroux M, Angriman I, Ruffolo C, Fiamingtlo P, Caglia P, Madia C, et al. Psoas abscess: a rare complication of Crohn's disease. Acta chir belg 2004;104:187-90.

2. Marin I, Serra I, Manosa M, Cabre E, Omenech E. Psoas abscess as a complication of Crohn's disease: report of three cases and literature review. Gastroenterol Hepatol 2009;32:557-61.

3. Shields D, Robinson P, Crowley TP. Iliopsoas abscess - a review and update on the literature. Inter J Surg 2012;10:466-9.

4. Koubaa M, Aouam A, Toumi A, Loussaief C, Brahim HB, Chakroun M. Psoas abscess as the first clinical manifestation of Crohn's disease. International Journal of Case Reports in Medicine, Vol. 2013 (2013), Article ID 507024, DOI: 10.5171/2013. 507024.

5. Agha FP, Woolsey E, Amendola MA. Psoas abscess in inflammatory bowel disease. Am J Gastro-enterol 1985;80:924-8.

6. Chern CH, Hu SC, Kao WF, Tsai J, Yen D, Lee CH. Psoas abscess: making an early diagnosis in the ED. AM J Emerg Med 1997;15:838.

7. Huang JJ, Ruaan MK, Lan RR, Wang MC. Acute pyogenic iliopsoas abscess in Taiwan: clinical features, diagnosis, treatments and outcome. J Infect 2000;40:248-55.

8. Richards RJ. Management of abdominal and pelvic abscess in Crohn's disease. WJGE 2011;3:209-12.

9. Ricci M, Meyer KK. Psoas abscess complicating Crohn's disease. A, J Gastroenterol 1985;80:970-7.

10. Femminineo AF, La Ban MM. Paraparesis in a patient with Crohn disease resulting from septic arthritis of the hip and psoas abscess. Arch Phys Med Rehabil 1988;69(3):223-5. 